\title{
Decentralized Dynamic Power Management with Local Information
}

\author{
Jing $\mathrm{Li}^{1}$, Guang Lin $^{2}$, Yu Huang ${ }^{3}$ \\ ${ }^{1}$ School of Information \& Electrical Engineering, Zhejiang University City College, \\ No. 48, Huzhou St., Hangzhou, China \\ ${ }^{2}$ Department of Mathematics, Purdue University, \\ 150 N. University Street, West Lafayette, USA \\ ${ }^{3}$ School of Electrical Engineering, Southeast University \\ Sipailou Campus of Southeast University, Nanjing, China \\ lijing@zucc.edu.cn
}

\begin{abstract}
The multi-period version of the optimal power flow can tackle the power dispatch problem of modern distribution system with distributed renewable energy sources and energy storage system. In this paper, a communication-efficient decentralized optimization algorithm (DOA) for the multi-period optimal power flow problem is presented. Firstly, the power management of modern distribution system is modelled as a linear conic optimization problem based on the conically relaxed power flow equations. Secondly, some ancillary variables at the junction bus are introduced to decompose the distribution system into several separate parts. Moreover, the DOA based on the extended version of Alternating Direction Method of Multipliers (ADMM) is proposed. The DOA evolves by partial local message exchanges without central coordination. Finally, the colouring scheme, which is in accord with the network colouring used in communication protocols to avoid packet collisions, is applied in the decentralized algorithm as well.
\end{abstract}

Index Terms-Distributed optimization; Optimal power flow; Renewable energy; Power management.

\section{INTRODUCTION}

The optimal power flow (OPF) has been marked as one of the most operational needs for a long time in the management of distribution systems. There are many algorithms used to solve the OPF problem, such as mathematical programming and heuristic optimization [1]. The development and utilization of renewable energy sources (RES) [2] brings great effect on modern distribution system. Both wind power and solar power based distributed generators (DGs) are of intermittent nature. On this account, technologies such as energy storage systems (ESS) and flexible demand [3], both of which contribute to increase the penetration of RES connecting to the power system as DGs.

The OPF with storage should consider some intertemporal constraints due to the operation of storage being strongly coupled over the time periods [4]. Therefore, the dynamic

Manuscript received 14 February, 2018; accepted 3 October, 2018.

This research was funded by Zhejiang Provincial Natural Science Foundation of China under Grant (No. LQ15F030001), Research Program of Department of Education of Zhejiang Province under Grant No. Y201635593. optimal power flow (DOPF) is developed to tackle the inter-temporal variables in the power management of modern distribution system with distributed ESS and DG [5], [6]. In modern intelligent distribution system, the distribution system operator is required to solve DOPF problem in decentralized manner to manage the huge increase in problem size [7]. The Alternating Direction Method of Multipliers (ADMM) is a promising approach for solving large-scale OPF [8]. In addition, various convexification and linearization have been proposed to simplify the OPF [9]. DC power flow equation is linearization method, however it has neither enough accuracy nor show what is happening in the line loss. It is worth noting that the nonlinear AC power flow constraints can be convexly relaxed [10]. Authors in [11], [12] have given some details about the convex relaxation of OPF. A second-order cone formulation for $\mathrm{AC}$ power flow problem is presented, so that the OPF is solved efficiently and accurately. A distributed semi-definite programming strategy for the OPF in the microgrids with DGs is proposed based on the bus injection model of power flow equations. A distributed algorithm for a second order cone relaxation of OPF over distribution system is designed. Authors in [13] have investigated the distributed optimization strategy for the DOPF, the network components were decomposed via the decomposition power of ADMM.

In this paper, the power management of modern distribution system with distributed ESS and DG is model as a DOPF problem. It is a multi-period version of the OPF solved over a finite horizon, thus it can optimize power across a network and across a time-horizon. Firstly, the non-linear and non-convex DOPF problem is converted into linear conic optimization problem by means of the conic relaxation of power flow equations. Then the distribution system is decomposed by introducing the ancillary variables at the junction bus of any two subsystems. Moreover, in order to solve the DOPF problem in decentralized manner, the communication-efficient decentralized optimization algorithm (DOA) based on the extended version of Alternating Direction Method of Multipliers (ADMM) is proposed. The colouring scheme is applied in the DOA as well. The DOA evolves by partial local message exchanges without central coordination. Finally, the DOA is applied to 
the DOPF problem of a test radial distribution system. The simulation results demonstrate the effectiveness of the proposed DOA and show the effect of the power management.

\section{PREliminary AND Notation}

Let a directed graph $\mathrm{G}=(\mathbf{E}(\mathrm{G}), \mathbf{\Omega}(\mathrm{G}))$ be the description of the radial distribution system, where $\mathbf{E}(\mathrm{G}):=\{0,1, \ldots, \mathrm{m}\}$ is the set of buses and $\boldsymbol{\Omega}(\mathrm{G})$ is the set of edges. The $j^{\text {th }}$ branch in the edge set $\boldsymbol{\Omega}(\mathrm{G})$ is expressed as $\psi_{j}:=(i, j)$ which means the line circuit from the parent bus $i$ to the child bus $j$ in the distribution system, where the bus $i$ is on the path from bus 0 (the root of the tree) to bus $j$.

In order to describe the relationship of edges and nodes in graph $\mathrm{G}$, we construct the induced incidence matrix $\mathbf{M}$ which is the $\{0, \pm 1\}$-matrix with rows and columns indexed by the vertices and edges respectively, that is

$$
(\mathbf{M})_{i j}= \begin{cases}1, & \text { if } i \text { is the child node of } \psi_{j}, \\ -1, & \text { if } i \text { is the parent node of } \psi_{j}, \\ 0, & \text { otherwise }\end{cases}
$$

where the root bus 0 is not considered in $\mathbf{M}$ and $\mathbf{M}$ is $\mathrm{m} \times \mathrm{m}$ matrix.

The main variables and assumptions are summarized in Table I. For every branch in the distribution system, the branch model of power flow equations can be build according to the Ohm's law and power balance at each bus [14]. Let $w_{j}:=$ $\left|V_{i}\right|^{2}+\left|I_{j}\right|^{2}$ and $l_{j}:=\left|V_{i}\right|^{2}-\left|I_{j}\right|^{2}$, thus the equations that describes the branch power flow can be rewritten as:

$$
\begin{gathered}
P_{j}-\sum_{k:(j, k) \in \mathbf{\Omega}(G)} P_{k}-R_{j} \frac{w_{j}-l_{j}}{2}=p_{j}, \\
Q_{j}-\sum_{k:(j, k) \in \mathbf{\Omega}(G)} Q_{k}-X_{j} \frac{w_{j}-l_{j}}{2}=q_{j}, \\
\frac{w_{j}+l_{j}}{2}-v_{j}-2\left(R_{j} P_{j}+X_{j} Q_{j}\right)+\left(R_{j}^{2}+X_{j}^{2}\right) \frac{w_{j}-l_{j}}{2}=0, \\
\left(P_{j}\right)^{2}+\left(Q_{j}\right)^{2}+\left(l_{j}\right)^{2}=\left(w_{j}\right)^{2},
\end{gathered}
$$

where $\forall(i, j) \in \mathbf{\Omega}(G)$,

$$
w_{1}+l_{1}=2\left|V_{0}\right|^{2}
$$

where $V_{0}$ denotes the voltage of the bus 0 . For a given nodal

\begin{tabular}{|c|c|}
\hline Symbols & Explanations \\
\hline & $\left(p_{j}^{\mathrm{dS}}(t)\right)$ of ESS on bus $j$ at time $t$ \\
\hline $\mathbf{R}, \mathbf{X}, \mathbf{C}$ & $\begin{array}{l}\text { the diagonal matrices in which the diagonal } \\
\text { elements are }(\mathbf{R})_{j j}=\mathbf{R}_{j},(\mathbf{X})_{j j}=X_{j},(\mathbf{C})_{j j}=\left(\mathbf{R}_{j}\right)^{2}+\left(X_{j}\right)^{2}\end{array}$ \\
\hline I, 0 & $\begin{array}{l}\text { the } m \times m \text { identity matrix and the } m \times m \text { zero matrix } \\
\text { in which all the elements are equal to zero }\end{array}$ \\
\hline $\mathbf{O}_{\mathrm{m}}$ & $\begin{array}{c}\text { the } m \times 1 \text { column vector in which all the elements } \\
\text { are equal to zero }\end{array}$ \\
\hline $\begin{array}{c}\boldsymbol{P}(t), \underset{\boldsymbol{w}(t)}{\boldsymbol{Q}(t), \boldsymbol{l}(t)} \boldsymbol{v}(t) \\
\end{array}$ & $\begin{array}{l}\text { the column vectors in which the elements are as } \\
\text { follows: }(\boldsymbol{P}(t))_{j}=P_{j}(t),(\boldsymbol{Q}(t))_{j}=Q_{j}(t),(\boldsymbol{v}(t))_{j}=\left|V_{j}(t)\right|^{2} \text {, } \\
\quad(\boldsymbol{w}(t))_{j}=\left|V_{i}(t)\right|^{2}+\left|I_{j}(t)\right|^{2},(\boldsymbol{l}(t))_{j}=\left|V_{i}(t)\right|^{2}-\left|I_{j}(t)\right|^{2}\end{array}$ \\
\hline $\begin{array}{c}\boldsymbol{p}(t), \boldsymbol{q}(t), \boldsymbol{p}^{\mathrm{D}}(t) \\
\boldsymbol{p}^{\mathrm{G}}(t), \boldsymbol{q}^{\mathrm{D}}(t) \\
\boldsymbol{q}^{\mathrm{G}}(t), \boldsymbol{p}^{\mathrm{cS}}(t) \\
\boldsymbol{p}^{\mathrm{dS}}(t)\end{array}$ & $\begin{array}{l}\text { the column vectors in which the elements are as } \\
\text { follows: }(\boldsymbol{p}(t))_{j}=p_{j}(t),(\boldsymbol{q}(t))_{j}=q_{j}(t),\left(\boldsymbol{p}^{\mathrm{D}}(t)\right)_{j}=p_{j}^{\mathrm{D}}(t), \\
\left(\boldsymbol{p}^{\mathrm{G}}(t)\right)_{j}=p_{j}^{\mathrm{G}}(t),\left(\boldsymbol{q}^{\mathrm{D}}(t)\right)_{j}=q_{j}^{\mathrm{D}}(t),\left(\boldsymbol{q}^{\mathrm{G}}(t)\right)_{j}=q_{j}^{\mathrm{G}}(t), \\
\left(\boldsymbol{p}^{\mathrm{cS}}(t)\right)_{j}=p_{j}^{\mathrm{cS}}(t),\left(\boldsymbol{p}^{\mathrm{dS}}(t)\right)_{j}=p_{j}^{\mathrm{dS}}(t)\end{array}$ \\
\hline
\end{tabular}
injected power $\left(p_{j}\right.$ and $\left.q_{j}\right)$, the branch power flow equations are the linear combination of the variables $\left(P_{j}, Q_{j}, v_{j}, l_{j}, w_{j}\right.$, $\forall(i, j) \in \mathbf{\Omega}(G)$ ) except for the quadratic equation (5).

TABLE I. NOTATIONS.

\begin{tabular}{|c|c|}
\hline Symbols & Explanations \\
\hline$I_{j}(t)$ & the complex current from bus $i$ to $j$ for $\psi_{j}$ at time $t$ \\
\hline$P_{j}(t)+\mathbf{j} Q_{j}(t)$ & the complex power flowing out from bus $i$ to $j$ at \\
\hline$V_{j}(t)$ & time $t$ \\
\hline$p_{j}(t)+\mathbf{j} q_{j}(t)$ & the complex voltage on bus $j$ at time $t$ \\
\hline$p_{j}{ }^{\mathrm{G}}(t)+\mathbf{j} q_{j} \mathrm{G}(t)$ & the complex power of DGs on bus $j$ at time $t$ \\
\hline$p_{j}{ }^{c \mathrm{~S}}(t), p_{j} \mathrm{dS}^{\mathrm{d}}(t)$ & the charging power $\left(p_{j}^{{ }_{j} \mathrm{~S}}(t)\right)$ and discharging power \\
\hline
\end{tabular}

\section{PROBLEM ForMULATION}

The proposed formulation of power management in the intelligent distribution system is a DOPF problem over a period $\mathbf{T}$, where $\mathbf{T}:=\left\{1,2, \ldots, t_{\text {end }}\right\}$. It minimizes the power losses and the deviation of the voltage across the whole time horizon subject to power flow constraints and system security constraints.

\section{A. Objective Function}

The objective function of the proposed DOPT includes two parts: one part $f_{j}^{L}(t)$ denotes the active power losses; and another part $f_{j}^{V}(t)$ describes the deviation of the voltage. Then the objective of the DOPF problem is minimize

$$
f_{0}=\sum_{t \in \mathbf{T}} \sum_{j \in \mathbf{\Omega}}\left\lfloor f_{j}^{L}(t)+f_{j}^{C}(t)\right\rfloor,
$$

where $\quad f_{j}^{L}(t)=\frac{R_{j}}{2} \times\left(w_{j}(t)-l_{j}(t)\right)$

and

$f_{j}^{V}(t)=\left(v_{j}(t)-v_{j m}\right)^{2}$. Here, $v_{j m}$ is the mean voltage of $j^{\text {th }}$ bus during the time horizon.

The objective function is a continuously differentiable convex function of the optimization variables for all $j \in \boldsymbol{\Omega}$ and $t \in \mathbf{T}$.

\section{B. Constraints}

1) Conically relaxed power flow constraints

Considering the branch power flow equalities mentioned above in (2)-(5), these equalities can be formulated by the intersection of the affine set and the second order cone

$$
\left[\begin{array}{ccccr}
\mathbf{M} & \mathbf{0} & \mathbf{0} & -\mathbf{R} / 2 & -\mathbf{R} / 2 \\
\mathbf{0} & \mathbf{M} & \mathbf{0} & -\mathbf{X} / 2 & -\mathbf{X} / 2 \\
2 \mathbf{R} & 2 \mathbf{X} & \mathbf{I} & \frac{-\mathbf{C}-\mathbf{I}}{2} & \frac{-\mathbf{C}+\mathbf{I}}{2}
\end{array}\right]\left[\begin{array}{c}
\boldsymbol{P}(\boldsymbol{t}) \\
\boldsymbol{Q}(\boldsymbol{t}) \\
\boldsymbol{v}(\boldsymbol{t}) \\
\boldsymbol{w}(\boldsymbol{t}) \\
\boldsymbol{l}(\boldsymbol{t})
\end{array}\right]=\left[\begin{array}{c}
\boldsymbol{p}(t) \\
\boldsymbol{q}(t) \\
\mathbf{o}_{\mathrm{m}}
\end{array}\right]
$$

where $\forall t \in \mathbf{T},(\boldsymbol{P}(t), \boldsymbol{Q}(t), \boldsymbol{v}(t), \boldsymbol{w}(t), \boldsymbol{l}(t)) \in \mathbf{K}(t)$.

$$
\mathbf{K}(t)=K_{1}(t) \times \ldots \times K_{m}(t),
$$

where $\forall t \in \mathbf{T}$, equality constraint (8) is the description of 
power flow equations (2)-(4), $\mathbf{M}$ is the induced incidence matrix in (1). And $\mathbf{K}_{j}(t)$ is the second order cone obtained by the conic relaxation of equations (5), that is

$$
\begin{gathered}
\mathbf{K}_{j}(t):=\left\{\left(P_{j}(t), Q_{j}(t), v_{j}(t), w_{j}(t), l_{j}(t)\right) \mid\right. \\
\left.w_{j}(t) \geq \sqrt{\left(P_{j}(t)\right)^{2}+\left(Q_{j}(t)\right)^{2}+\left(l_{j}(t)\right)^{2}}\right\} .
\end{gathered}
$$

\section{2) Security constraints}

For the first branch that points from bus 0 to bus 1 , bus 0 denotes the substation bus that has a fixed voltage $V_{0}$

$$
(l(t))_{1}+(w(t))_{1}=2\left|V_{0}\right|^{2}
$$

where $\forall t \in \mathbf{T}$. Branch capacity requires that the branch current will not be allowed to go above a threshold

$$
\boldsymbol{w}(t)-\boldsymbol{l}(t) \preceq 2\left|I_{\max }\right|^{2}
$$

where $\forall t \in \mathbf{T}, I_{\max }$ is the upper bound of the branch current.

The voltage magnitudes must be maintained in tight ranges

$$
\left|V_{\min }\right|^{2} \preceq \boldsymbol{v}(t) \preceq\left|V_{\max }\right|^{2}
$$

where $\forall t \in \mathbf{T}, V_{\min }$ and $V_{\max }$ are the lower and upper bound of the bus voltage.

\section{3) Storage system constraints}

The generated power is determined by the generated power by the renewable energy based DGs and the state of charge of ESS. For the $j$ th bus

$$
p(t)=p^{D}(t)+p^{c S}(t)-p^{d S}(t)-p^{G}(t)
$$

where $\forall t \in \mathbf{T}$. Suppose that the output power of the solar-based DGs follow the point from maximum-power point tracking. The active power of DGs is suggested to be made maximal use of, that is, $\boldsymbol{p}^{\boldsymbol{G}}(t)$ can't be controlled. However, the ESS can be considered as either a generator when it is discharged or a load when it is charged. Then the charging and discharging power of ESS, $\boldsymbol{p}^{c S}(t)$ and $\boldsymbol{p}^{d S}(t)$, can be controlled and optimized.

At $j$ th bus in the distribution system, let $E_{j}(t)$ denote the amount of energy storage at each time $t \in \mathbf{T}$. The amount of storage at time $t$ depends on the residual energy at the last time $t-1$ and the rate of charge/discharge of energy at time $t$ [15]. That is

$$
\begin{aligned}
E_{j}(t) & =E_{j}(t-1)+\Delta t \times \frac{\left(p^{c S}(t)\right)_{j}}{\eta_{c}}- \\
& -\Delta t \times \eta_{d} \times\left(p^{d S}(t)\right)_{j},
\end{aligned}
$$

where $\forall \psi_{j} \in \mathbf{\Omega}(G), \forall t \in \mathbf{T} \eta_{c}$ and $\eta_{d}$ denote the charge and discharge efficiency respectively, $\Delta t$ denotes the length of time step from $t-1$ to $t$, and $E_{j}(0)=E_{I N}$ denotes the initial condition of energy level in ESS at the beginning.

The ESS is not an energy source but an assistant storage device, so the power generated by the ESS is the power that has been accumulated in the ESS for a time. Since a time horizon (e.g. $t_{\mathrm{end}}=24 \mathrm{~h}$ ) is considered for optimization, energy level in the storage device at the final time point should be returned to the initial state [16]

$$
E_{j}(0)=E_{j}\left(t_{\text {end }}\right)
$$

where $\forall \psi_{j} \in \mathbf{\Omega}(G)$. Moreover, the amount of energy in the storage device should be bounded as

$$
E_{j, \min } \leq E_{j}(t) \leq E_{j, \max }
$$

where $\forall \psi_{j} \in \mathbf{\Omega}(G), \forall t \in \mathbf{T},\left(E_{j, \min }, E_{j, \max }\right)$ denotes the lower and upper bounds on energy level at $j$ th bus. Here, it is assumed that they are $20 \%$ and $90 \%$ of the installed capacity of the storage units, respectively.

Considering the effect on the cycle life of storage device, the charge/discharge power should be capped by the battery's nominal charge/discharge rate by:

$$
\begin{aligned}
& 0 \leq\left(p^{c S}(t)\right)_{j} \leq p_{j}^{S r}, \\
& 0 \leq\left(p^{d S}(t)\right)_{j} \leq p_{j}^{S r},
\end{aligned}
$$

where $\forall \psi_{j} \in \mathbf{\Omega}(G), \forall t \in \mathbf{T}, p_{j}^{S r}$ denotes nominal charging rate of battery at $j$ th bus, and $-p_{j}^{S r}$ denotes the discharging rate.

$$
\text { Let }\left(p^{c S}(t)\right)_{j} \times\left(p^{d S}(t)\right)_{j}=0 \text { indicates that either the }
$$

charging or discharging process of the ESS at time $t$, there can only be one process at any one time. Using the McCormick Envelopes [17], the nonlinear constraint can be relaxed by:

$$
\left\{\begin{array}{l}
0 \geq\left(p^{c S}(t)\right)_{j} \times p_{j}^{S r}+\left(p^{d S}(t)\right)_{j} \times p_{j}^{S r}-p_{j}^{S r} \times p_{j}^{S r}, \\
0 \leq\left(p^{c S}(t)\right)_{j} \times p_{j}^{S r}, \\
0 \leq\left(p^{d S}(t)\right)_{j} \times p_{j}^{S r},
\end{array}\right.
$$

where $\forall \psi_{j} \in \mathbf{\Omega}(G), \forall t \in \mathbf{T}$.

4) Reactive power constraints

The penetration of solar-based DGs can do inverter-based reactive power control [18]. So the reactive power of DGs can be controllable and optimized:

$$
\begin{gathered}
\boldsymbol{q}(t)=\boldsymbol{q}^{\boldsymbol{D}}(t)-\boldsymbol{q}^{\boldsymbol{G}}(t), \\
\boldsymbol{q}^{\boldsymbol{G} \boldsymbol{m i n}}(t) \preceq \boldsymbol{q}^{\boldsymbol{G}}(t) \preceq \boldsymbol{q}^{\boldsymbol{G m a x}}(t),
\end{gathered}
$$

where $\forall t \in \mathbf{T}, \boldsymbol{q}^{\boldsymbol{G m i n}}(t)$ and $\boldsymbol{q}^{\boldsymbol{G m a x}}(t)$ denote the lower and upper bounds of the injected reactive powers of DGs at time $t$. 


\section{Dynamic Optimal Power Flow Problem in Centralized} Formulation

Given

$$
\boldsymbol{y}:=\left(\boldsymbol{y}_{j}(t), \forall \psi_{j} \in \mathbf{\Omega}(G), \forall t \in \mathbf{T}\right),
$$

where $\boldsymbol{y}_{j}(t):=\left(P_{j}(t), Q_{j}(t), v_{j}(t), w_{j}(t), l_{j}(t), p_{j}^{c S}(t)\right.$, $\left.p_{j}^{d S}(t), q_{j}^{G}(t)\right)$.It should be noted that the feasible set of the DOPF problem, $\boldsymbol{D}:=\{(\boldsymbol{y}) \mid \boldsymbol{y}$ satisfies (8)-(18) $\}$, is convex.

Consider the DOPF in centralized formulation:

$$
\text { DOPF-c: } \min _{\boldsymbol{y}} f_{0}(y) \text {, subject to } y \in \mathbf{D} \text {. }
$$

Clearly, this problem is belonging to the conic programming who's the feasible set is the intersection of affine set and second order cone. And the optimal solution can give a true statement when it is substituted into (2)-(6).

\section{DECENTRALIZED OPTIMIZATION ALGORITHM BASED ON LOCAL MESSAGE}

\section{A. Decomposition}

It can be seen from Fig. 1 that the decomposition is happened in junction bus $n_{k}$. Before decomposition, the graph $\mathrm{G}$ is consist of two sub-graph $\mathrm{G}_{a}$ and $\mathrm{G}_{b}$, where the edge sets of the sub-graphs satisfy $\boldsymbol{\Omega}\left(\mathrm{G}_{a}\right) \cup \boldsymbol{\Omega}\left(\mathrm{G}_{b}\right)=\boldsymbol{\Omega}(\mathrm{G})$, $\boldsymbol{\Omega}\left(\mathrm{G}_{a}\right) \cap \boldsymbol{\Omega}\left(\mathrm{G}_{b}\right)=\emptyset$ and the node sets satisfy $\mathbf{E}\left(\mathrm{G}_{a}\right) \cup \mathbf{E}\left(\mathrm{G}_{b}\right)=$ $\mathbf{E}(\mathrm{G}), \mathbf{E}\left(\mathrm{G}_{a}\right) \cap \mathbf{E}\left(\mathrm{G}_{b}\right)=k$. To separate the network, the dummy buses $n_{k+}$ and $n_{k-}$ are introduced such that the distribution system is separated into the receiving and sending parts. Thus the generated graph $\mathrm{G}_{a}^{\prime}$ and $\mathrm{G}_{b}^{\prime}$, of which edge sets are $\boldsymbol{\Omega}\left(\mathrm{G}_{a}^{\prime}\right)$ $=\boldsymbol{\Omega}\left(\mathrm{G}_{a}\right), \boldsymbol{\Omega}\left(\mathrm{G}_{b}^{\prime}\right)=\boldsymbol{\Omega}\left(\mathrm{G}_{b}\right)$ and node sets $\left(\mathbf{E}\left(\mathrm{G}_{a}^{\prime}\right)\right.$ and $\left.\mathbf{E}\left(\mathrm{G}_{b}^{\prime}\right)\right)$ are obtained by replacing the common node $k$ with $k+$ and $k$ in $\mathbf{E}\left(\mathrm{G}_{a}\right)$ and $\mathbf{E}\left(\mathrm{G}_{b}\right)$ respectively, are used to describe the receiving and sending parts [19].

In the optimization problem DOPF-c, the variable $\boldsymbol{y}$ falls into two categories: the core variables of the separate parts, and their common variables. Suppose that the decomposition is happened in the junction bus $n_{k}$, the core variables $\boldsymbol{y}_{a}$ and $\boldsymbol{y}_{b}$ are given by:

$$
\left\{\begin{array}{l}
\boldsymbol{y}_{a}:=\left(\boldsymbol{y}_{j}(t), \psi_{j} \in \boldsymbol{\Omega}\left(G_{a}\right) /\left\{\psi_{k}\right\}, t \in \mathbf{T}\right), \\
\boldsymbol{y}_{b}:=\left(\boldsymbol{y}_{j}(t), \psi_{j} \in \mathbf{\Omega}\left(G_{b}\right) /\left\{\psi_{h}\right\}, t \in \mathbf{T}\right) .
\end{array}\right.
$$

The common variables consist of the voltage in junction bus $n_{k}$ and the branch power in the branch $\psi_{h}=(k, h)$, thus the common variables are described by $\boldsymbol{y}_{a b}=\left(v_{k}(t), P_{h}(t), Q_{h}(t)\right.$, $t \in \mathbf{T})$.

Then the optimal variable $\boldsymbol{y}=\left(\boldsymbol{y}_{a}, \boldsymbol{y}_{a b}, \boldsymbol{y}_{b}\right)$ can be obtained by the following:

$$
\begin{gathered}
\text { DOPF-cr: } \min _{\left(\boldsymbol{y}_{a}, \boldsymbol{y}_{a b}, \boldsymbol{y}_{b}\right)} f_{0 a}\left(y_{a}\right)+f_{0 b}\left(y_{b}\right), \\
\text { subject to }\left(\boldsymbol{y}_{a}, \boldsymbol{y}_{a b}, \boldsymbol{y}_{b}\right) \in \mathbf{D},
\end{gathered}
$$

where

$$
f_{0 a}=\sum_{t \in \mathbf{T}} \sum_{j \in \mathbf{\Omega}\left(G_{a}\right)}\left\lfloor f_{j}^{L}(t)+f_{j}^{V}(t)\right\rfloor
$$

$$
f_{0 b}=\sum_{t \in \mathbf{T}} \sum_{j \in \boldsymbol{\Omega}\left(G_{b}\right)}\left\lfloor f_{j}^{L}(t)+f_{j}^{V}(t)\right\rfloor .
$$

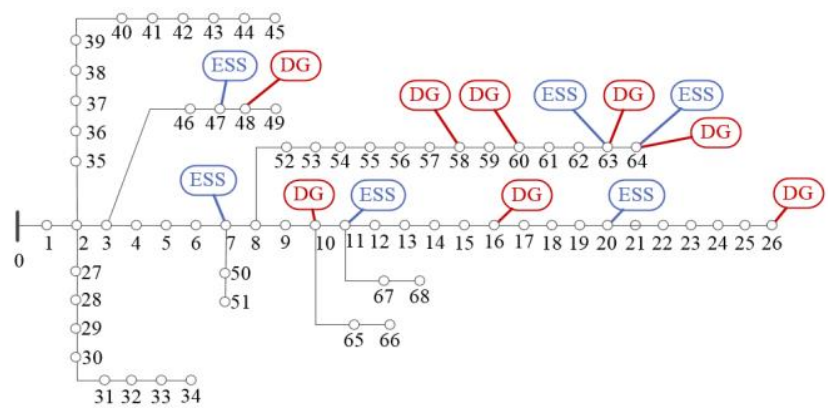

Fig. 1. The PG\&E 69-buses distribution system with DGs

The objective function $f_{0}(\boldsymbol{y})$ of problem DOPF-c is independent of the common variable $\boldsymbol{y}_{a b}$, so $f_{0}=f_{0 a}+f_{0 b}$. The constraints couple the core and common variables together.

For the sending part, $P_{h}(t)+\mathbf{j} Q_{h}(t)$ is regarded as the dummy load power at the dummy node $n_{k+}$. So the net active and reactive power at the node $n_{k+}$ is equal to the original net power at node $n_{k}$ plus the dummy load power, that is:

$$
\begin{aligned}
& p_{k+}(t)=P_{h}(t)+p_{k}(t), \\
& q_{k+}(t)=\mathrm{Q}_{h}(t)+q_{k}(t),
\end{aligned}
$$

where $\forall t \in \mathbf{T}$, for the receiving part, the dummy node $n_{k^{+}}$can be considered as a dummy substation whose voltage $v_{k-}(t)=$ $v_{k}(t)$. In addition, the voltage of dummy substation satisfies

$$
v_{k-}(t)=\frac{w_{h}(t)+l_{h}(t)}{2},
$$

where $\forall t \in \mathbf{T}$. For the sake of decomposition, the auxiliary variables $\left(p_{k+}^{D}(t), q_{k+}^{D}(t)\right)$ are introduced into the sub-problem of the sending part $G_{a}^{\prime}$. Meanwhile, the auxiliary variable $v_{k-(t)}$ is introduced into the sub-problem of the receiving part $G_{b}^{\prime}$. The auxiliary variables $\left(v_{k-}(t), p_{k+}^{D}(t)\right.$ ,$\left.q_{k+}^{D}(t)\right)$ can be regard as the copy of the common variables $\boldsymbol{y}_{a b}$.

The introduction of the auxiliary variables, that is to say, copies for common variables are given to the separate parts respectively. So with help of the auxiliary variables, the common variable $\boldsymbol{y}_{a b}$ is decomposed into two variables:

$$
\left\{\begin{array}{c}
z_{a}^{(a, b)}:=\left(v_{k}(t), p_{k+}^{D}(t), q_{k+}^{D}(t), t \in \mathbf{T}\right), \\
z_{b}^{(b, a)}:=\left(v_{k-}(t), P_{h}(t), Q_{h}(t), t \in \mathbf{T}\right) .
\end{array}\right.
$$

The problem DOPF-c can be reformulated by:

$$
\text { DOPF-cd: } \min _{\left(\boldsymbol{y}_{\boldsymbol{a}}, z_{a}^{(a, b)}, \boldsymbol{y}_{\boldsymbol{b}}, z_{a}^{(b, a)}\right)} f_{0 a}\left(y_{a}\right)+f_{0 b}\left(y_{b}\right),
$$




$$
\begin{gathered}
\text { s.t. }\left\{\begin{array}{l}
\left(\boldsymbol{y}_{a}, z_{a}^{(a, b)}\right) \in \mathbf{D}_{a}, \\
\left(\boldsymbol{y}_{b}, z_{b}^{(b, a)}\right) \in \mathbf{D}_{b},
\end{array}\right. \\
\boldsymbol{z}_{a}^{(a, b)}=\boldsymbol{z}_{b}^{(b, a)},
\end{gathered}
$$

where the equality constraint (34) is to make sure that the copes are equivalent. Two sub-feasible sets $\mathbf{D}_{a}$ and $\mathbf{D}_{b}$ are with respect to the part $G_{a}^{\prime}$ and $G_{b}^{\prime}$, they are given by:

$$
\left\{\begin{array}{c}
\mathbf{D}_{a}=\left\{\left(\boldsymbol{y}_{a}, \boldsymbol{z}_{a}^{(a, b)}\right) \mid\left(\boldsymbol{y}_{a}, \boldsymbol{z}_{a}^{(a, b)}\right) \text { satisfy (8) (17),(18),(19) }\right\} \\
\mathbf{D}_{b}=\left\{\left(\boldsymbol{y}_{b}, \boldsymbol{z}_{b}^{(b, a)}\right) \mid\left(\boldsymbol{y}_{b}, \boldsymbol{z}_{b}^{(b, a)}\right)\right. \text { satisfy (8) (17),(20)\}. }
\end{array}\right.
$$

The problem DOPF-cd with the coupling constraint (34) can be decoupled into two sub-problems based on dual decomposition. By relaxing the coupling constraint as:

$$
\begin{gathered}
\min _{\boldsymbol{y}_{\boldsymbol{a}}, z_{a}^{(a, b)}, \boldsymbol{y}_{\boldsymbol{b}}, z_{a}^{(a, b)}} f_{0 a}\left(\boldsymbol{y}_{a}\right)+f_{0 b}\left(\boldsymbol{y}_{b}\right)+ \\
+\mu^{T}\left(\boldsymbol{z}_{a}^{(a, b)}-\boldsymbol{z}_{b}^{(b, a)}\right), \\
\text { subject to }\left\{\begin{array}{l}
\left(\boldsymbol{y}_{a}, \boldsymbol{z}_{a}^{(a, b)}\right) \in \mathbf{D}_{a}, \\
\left(\boldsymbol{y}_{b}, \boldsymbol{z}_{b}^{(b, a)}\right) \in \mathbf{D}_{b},
\end{array}\right.
\end{gathered}
$$

where $\mu$ is the Lagrange multiplier corresponding to the constraint (34).

\section{B. Decentralized Algorithm}

Supposing that the distribution system $\mathrm{G}$ is divided into parts $\left\{G_{g}^{\prime}=\left(\mathbf{E}_{g}, \boldsymbol{\Omega}_{g}\right), g=1, \ldots, n.\right\}$, each part is considered as a communication node in the corresponding $\mathrm{CN}$ is $G_{c}=\left(\mathbf{E}_{c}\right.$, $\boldsymbol{\Omega}_{c}$ ), where $\mathbf{E}_{c}=\left\{c_{1}, \ldots, c_{n}\right\}$ is the set of nodes and $\boldsymbol{\Omega}_{c} \subseteq \mathbf{E}_{c} \times$ $\mathbf{E}_{c}$ is the set of edges.

Let $z_{g}=\left\{z_{g}^{(g, h)}\right\}_{\left(c_{g}, c_{h}\right) \in \boldsymbol{\Omega}_{c}}$ be the set of the auxiliary variables in sub-graph $G_{g}^{\prime}$, where vector $z_{g}^{(g, h)}$ denotes the data (include voltage and branch power) to be transferred from communication node $c_{g}$ to $c_{h}$ via the communication link $\left(c_{g}, c_{h}\right) \in \boldsymbol{\Omega}_{c}$. Let $\boldsymbol{y}_{g}$ be the core variable of the separate part $G_{g}^{\prime}$.

Thus the problem DOPF-c can be rewritten as:

$$
\begin{aligned}
& \text { DOPF-cdn: minimize } f_{01}\left(y_{1}\right)+ \\
& +f_{02}\left(y_{2}\right)+\ldots+f_{0 n}\left(y_{n}\right), \\
& \text { s.t. }\left(\boldsymbol{y}_{g}, \boldsymbol{z}_{g}\right) \in D_{g},
\end{aligned}
$$

where $g=1, \ldots, n$.

$$
\left\{\begin{array}{l}
z_{g}^{(g, h)}=z_{h}^{(h, g)}, \\
\forall\left(c_{g}, c_{h}\right) \in \mathbf{\Omega}_{C},
\end{array}\right.
$$

where $f_{0 g}\left(y_{g}\right)=\sum_{t \in \mathbf{T}} \sum_{g \in \boldsymbol{\Omega}_{g}}\left\lfloor f_{j}^{L}(t)+f_{j}^{C}(t)\right\rfloor$, and $\boldsymbol{D}_{g}$ is the $g$-th feasible set of part $G_{g}^{\prime}$.

The above problem DOPF-cdn is now separable except for the coupled equality constraints (40). It should be note that all the feasible sets $\left\{D_{g}, g=1, \ldots, n\right\}$ are convex. As in the method of multipliers, the augmented Lagrangian of problem DOPF-cdn is formed

$$
\begin{array}{r}
L_{\rho}\left(\boldsymbol{y}_{1}, \boldsymbol{z}_{1}, \cdots, \boldsymbol{y}_{n}, \boldsymbol{z}_{n}, \lambda\right)=\sum_{g=1}^{n} f_{0 g}\left(\boldsymbol{y}_{g}\right)+ \\
+\sum_{\left(c_{g}, c_{h}\right) \in \mathbf{\Omega}_{c}}\left(\begin{array}{c}
\lambda_{\boldsymbol{g}, \boldsymbol{h}}^{\mathbf{T}}\left(\boldsymbol{z}_{g}^{(g, h)}-\boldsymbol{z}_{h}^{(h, g)}\right)+ \\
+\frac{\rho}{2}\left\|\boldsymbol{z}_{g}^{(g, h)}-\boldsymbol{z}_{h}^{(h, g)}\right\|_{2}^{2}
\end{array}\right),
\end{array}
$$

where the $\lambda=\left\{\boldsymbol{\lambda}_{\boldsymbol{g}, \boldsymbol{h}}\right\}_{\left(c_{g}, c_{h}\right) \in \mathbf{\Omega}_{c}}$ is the set of the dual variables, and $\rho>0$.

The decentralized optimization algorithm of ADMM includes the iterations:

$$
\begin{gathered}
\left(\boldsymbol{y}_{1}{ }^{k+1}, z_{1}{ }^{k+1}\right)= \\
=\underset{\left(\boldsymbol{y}_{1}, z_{1}\right) \in D_{1}}{\arg \min } L_{\rho}\left(\boldsymbol{y}_{1}, \boldsymbol{z}_{1}, \boldsymbol{y}_{2}{ }^{k}, z_{2}{ }^{k}, \ldots, \boldsymbol{y}_{n}{ }^{k}, \boldsymbol{z}_{n}{ }^{k}, \lambda^{k}\right), \\
\vdots \\
\left(\boldsymbol{y}_{n}{ }^{k+1}, \boldsymbol{z}_{n}{ }^{k+1}\right)= \\
=\underset{\left(\boldsymbol{y}_{n}, z_{n}\right) \in D_{n}}{\arg \min } L_{\rho}\left(\boldsymbol{y}_{1}^{k+1}, \boldsymbol{z}_{1}^{k+1}, \boldsymbol{y}_{2}^{k+1}, \boldsymbol{z}_{2}^{k+1}, \ldots, \boldsymbol{y}_{n}, \boldsymbol{z}_{n}, \lambda^{k}\right), \\
\lambda_{g, h}^{k+1}=\lambda_{g, h}^{k}+\rho\left(\boldsymbol{z}_{g}{ }^{k, h), k+1}-\boldsymbol{z}_{h}^{(h, g), k+1}\right),
\end{gathered}
$$

where $\forall\left(c_{g}, c_{h}\right) \in \mathbf{\Omega}_{C}$. Furthermore, the network colouring scheme [20] is used to synchronize the order of the nodes. Each communication node is assigned a colour in $C=\{1, \ldots$, $C$, where $C:=|\boldsymbol{C}|$ is the total number of colours, and $\boldsymbol{C}\left(c_{k}\right)$ denotes the colour of node $c_{k}$. The colour scheme requires $\boldsymbol{C}\left(c_{k}\right) \neq \boldsymbol{C}\left(c_{h}\right)$ for all $\left(c_{k}, c_{h}\right) \in \boldsymbol{\Omega}_{c}$. Up on that each iteration is divided into $C$ steps. In each step, all the communication nodes with the same colour perform the optimizations in parallel.

The proposed algorithm is shown in Algorithm 1. Firstly the initialization of the auxiliary variables includes: randomly choosing the auxiliary voltages, the auxiliary powers and the dual variables. Significantly, the value of the dual variables must satisfy $\lambda_{g, h}^{k}=-\lambda_{h, g}^{k}$ for $\left(c_{g}, c_{h}\right) \in \mathbf{\Omega}_{C}$.

In steps $2-6$, the communication nodes work according to their colours, and the nodes with the same colour solve the optimization subsystem in parallel. In step 4, the solution of the optimization subsystem $\left(\boldsymbol{y}_{g}{ }^{k+1}, \boldsymbol{z}_{g}{ }^{k+1}\right)$ can be calculated by 


$$
\begin{gathered}
\min _{\left(\boldsymbol{y}_{g}, \boldsymbol{z}_{g}\right) \in D_{g}} f_{0 g}\left(\boldsymbol{y}_{g}\right)+ \\
+\sum_{j \in E s_{g}}\left(-\left(\lambda_{j, g}^{k}\right)^{\mathbf{T}} \boldsymbol{z}_{g}^{(g, j)}+\frac{\rho}{2}\left\|\boldsymbol{z}_{g}^{(g, j)}-\boldsymbol{z}_{j}^{(j, g), k+1}\right\|_{2}^{2}\right)+ \\
+\sum_{h \in E r_{g}}\left(\left(\lambda_{g, h}^{k}\right)^{\mathbf{T}} \boldsymbol{z}_{g}^{(g, h)}+\frac{\rho}{2}\left\|\boldsymbol{z}_{g}^{(g, h)}-\boldsymbol{z}_{h}^{(h, g), k}\right\|_{2}^{2}\right)
\end{gathered}
$$

where $E s_{g}$ is the set of sending subsystems of subsystem $g$ and $\mathrm{Er}_{\mathrm{g}}$ is the set of receiving subsystems of subsystem $g$.

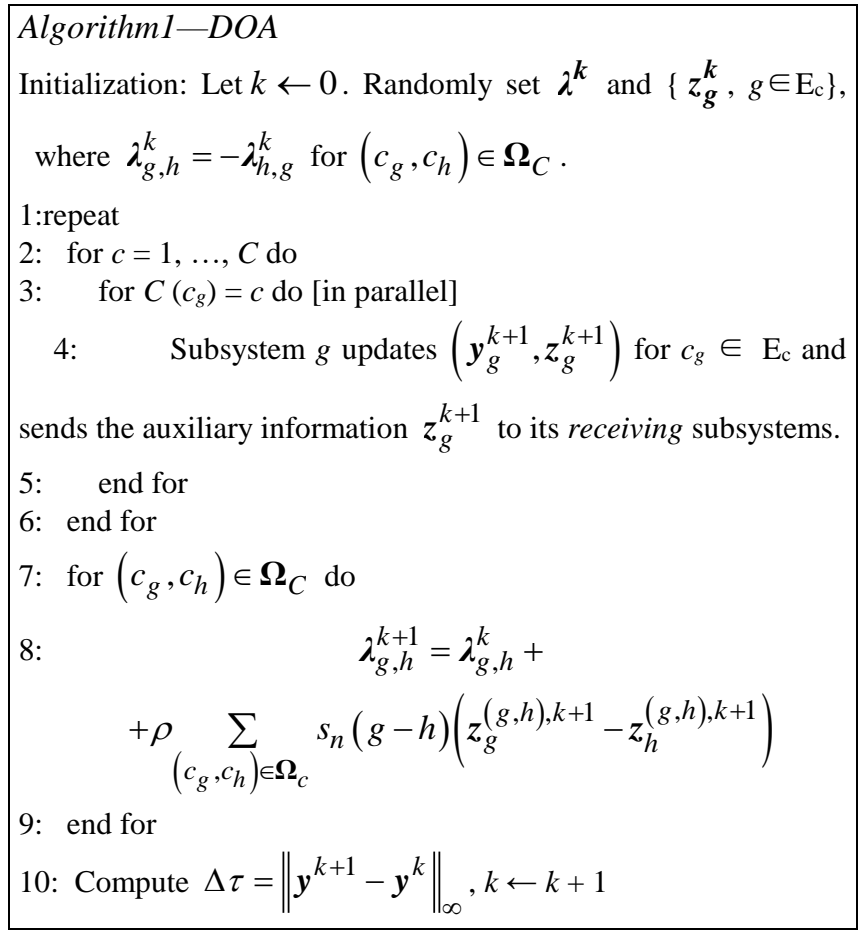

Then the dual variables are updated in steps 7-9. Here the function $s_{n}()$ is the sign function, defined as $s_{n}(x)=1$ if $x>0$, and $s_{n}(x)=-1$ if $x<0$.

Finally, the error $\Delta \tau=\left\|\boldsymbol{y}^{k+1}-\boldsymbol{y}^{k}\right\|_{\infty}$ is calculated and $k \leftarrow k+1$ is set, the process is repeated until convergence. It implies that the algorithm is convergent when $\Delta \tau$ converges to zero. It can be easily proved that the proposed algorithm DOA is convergent since the $\left\{D_{g}, g=1, \ldots, n\right\}$ are convex and the communication network $G_{c}$ is connected [21], [22].

\section{CASE STUdy}

The distribution system considered for the case study is the PG\&E 69-buses benchmark examples. As shown in Fig. 1, it is a $12.66 \mathrm{kV}$ distribution system with a peak load of $3802.19+\mathrm{j} 2694.60 \mathrm{kVA}$. The main substation at bus 0 is used to feed an area. Moreover, the data of the system are given in [23].

There are $200 \mathrm{~kW}$ solar based-DGs connected to 10,16 and 26 respectively; and $500 \mathrm{~kW}$ solar based-DGs connected to 48, 58, 60, 63 and 64 respectively; and $200 \mathrm{kWh}$ ESSs connected to 7, 11, 20, 47, 63 and 64 respectively.

In order to improve the life and efficiency of the storage device, the current flowing through the ESS must be limited within a certain rage. Then the maximum absorbable and available power must be considered, the suggested limit is $1 \mathrm{C}$ in the charging phase.

$$
p_{j}^{S r}=C_{b} \times 1,
$$

where $C_{b}$ denotes the capacity of ESS in $\mathrm{kWh}$. And the initial amount of energy in the ESS is set to be sixty percent of capacity, $E(0)=0.6 C_{b}$.

Let us discretize the day into 24 uniform time intervals, each of which is equal to 1 hour ( $\Delta t=1$ hour). Figure 2 shows the average power demand and the average solar power of a day. The hourly power data for the system under study is as a percentage of the annual peak value. It can be seen that the output power of solar-based DG reaches the peak at midday, nevertheless, there is no solar power when the sun goes down. The energy storage system is necessary that it can provide power and store the surplus power as well.

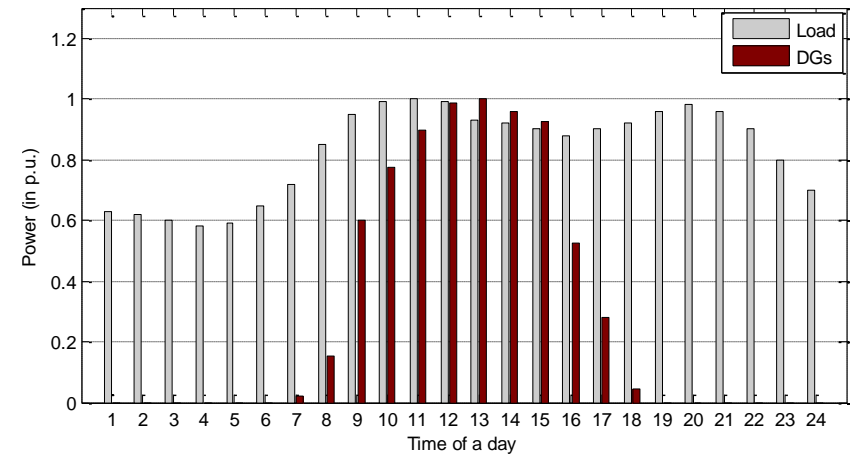

Fig. 2. Hourly average load and power generated by solar based-DG of a day.

The following scenarios are used to illustrate the effectiveness of the DOA algorithm and the DOPF model.

1. No DG and ESS installed in the distribution system. No other distributed energy devices are considered.

2. As for scenario $1+$ DG. The location and capacity of solar-based DGs are shown in the Table II. No reactive power generated by DGs.

3. As for scenario $2+$ ESS. The location and capacity of ESSs are given in the Table II. Using the proposed DOA to optimal dispatch active power of ESS and reactive power of DGs.

\section{A. Implementation}

The dynamic power flow optimization model in distribution system have been programmed in MATLAB R2010b; the proposed distributed algorithm DOA has been implemented by MOSEK's optimization solver. The experiments are conducted on a 64-bit PC with Intel Core 17 CPU at $1.73 \mathrm{GHz}$ and 4GB RAM.

\section{B. Results}

For this 69-buses distribution system, the developed communication-efficient DOA can give the optimal active and reactive power management at the bus which has DGs and ESS in fully distributed manner. The minimum bus voltage of the system for three scenarios is shown in Fig. 3. With the help of the power generated by solar-based DGs, the voltage drop is avoided when the load reaches peak level at midday. In addition, the charging and discharging management of installed ESSs rise the voltage in the evening, and smooth the 
voltage profile as well. In addition, reactive power of DGs improve the voltage in the evening. Note that smother voltage profiles are obtained when energy storage systems are enabled in the third scenario (shown by the blue solid line).

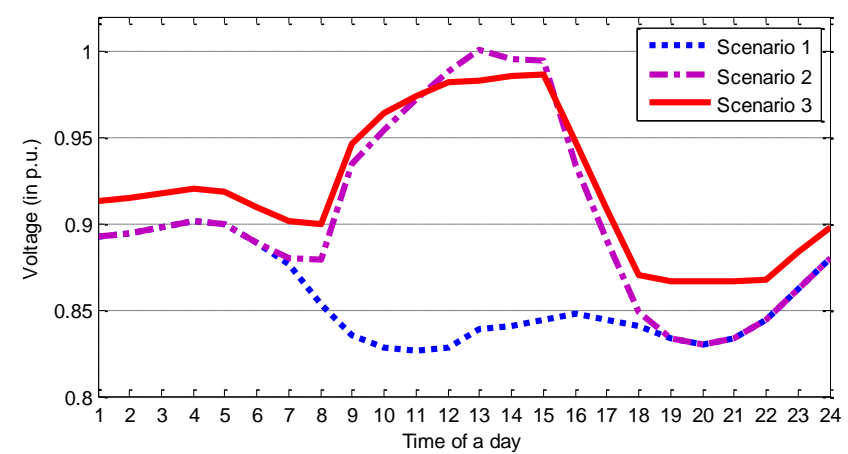

Fig. 3. Minimum bus voltage profile for different scenarios.

Figure 4 shows the hourly power losses for different scenarios. It can be observed that the power loss of the distribution system is significantly decreased at noon and in the evening in the third scenario. The total power losses during a day for different scenarios are summarized in Table II. The power loss is $3777.99 \mathrm{~kW}$ without the DG and ESS, the addition of DGs can decrease the power loss by $1189.07 \mathrm{~kW}(31.48 \%)$. In terms of the proposed DOA, optimal management of ESSs' active power and DGs' reactive power can further reduce the power loss by $758.48 \mathrm{~kW}(20.08 \%)$.

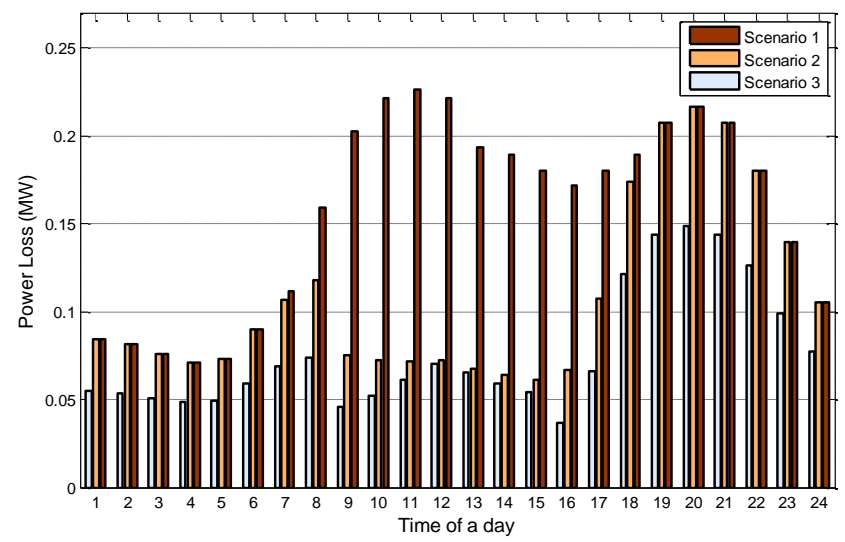

Fig. 4. The hourly power losses of the system for different scenarios.

TABLE II. THE TOTAL POWER LOSSES DURING A DAY FOR DIFFERENT SCENARIOS

\begin{tabular}{|c|c|c|c|}
\hline & Scenario 1 & Scenario 2 & Scenario 3 \\
\hline Power loss & $3777.99 \mathrm{~kW}$ & $2588.92 \mathrm{~kW}$ & $1830.44 \mathrm{~kW}$ \\
\hline
\end{tabular}

Accordingly, it can smooth the voltage profile and decrease the power losses if the intelligent distribution system optimally manages the power of DGs and ESSs. The ESS devices are located at the same bus as DGs and is able to directly manage the fluctuated renewable energy. The operating schedule for the ESS devices on different buses are shown in Fig. 5. The ESSs are in the charging process when the curves are above the zero level. Otherwise, the ESSs are in the discharging process when the curves are below the zero level. It can be seen from the figure that the ESS devices can absorb the surplus energy when the power generated by the solar-based DG reached peak level at midday. However, during the periods when there is no solar power, the ESS devices generate power.

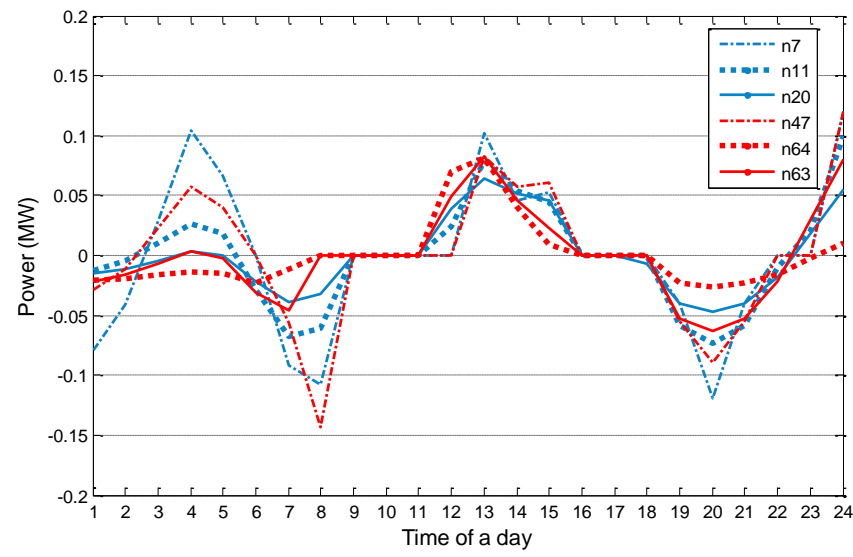

Fig. 5. The hourly output power of ESS on corresponding buses.

Figure 6 shows the relative error $\left\|\left(\boldsymbol{X}^{k}-\boldsymbol{X}^{*}\right) / \boldsymbol{X}^{*}\right\|_{\infty}$ versus the number of iterations. Here, $\boldsymbol{X}^{k}$ is the solution of DAO-algorithm at the end of $k$ th iteration, and $\boldsymbol{X}^{*}$ is the optimal solution of DOPF-c, computed in a centralized way.

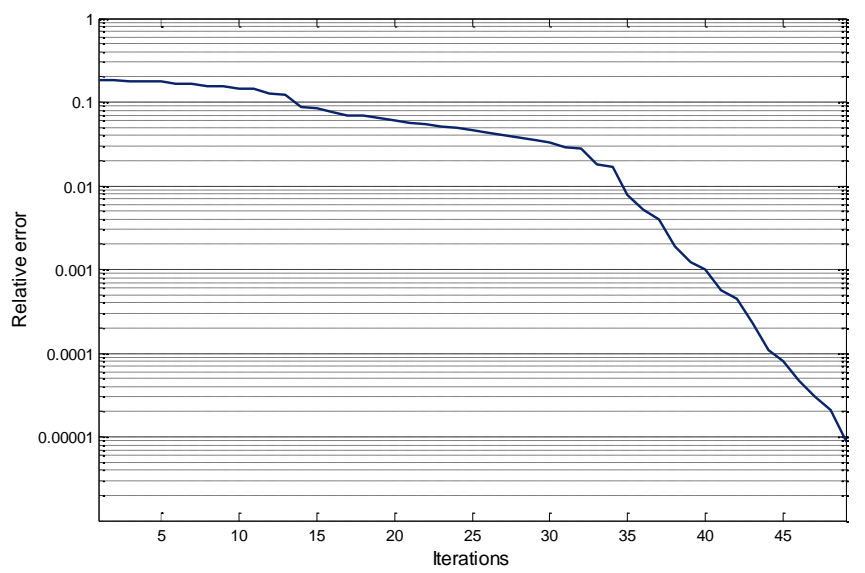

Fig. 6. The relative error versus the iterations.

The figure shows that the proposed DAO-algorithm requires about 40 iterations to achieve any relative error bellow $10^{-3}$. Moreover, subsystems only talk to their neighbours for the information, and the exchange information is the part that is related to the neighbours. It can be concluded that the DAO-algorithm is convergent and efficient.

\section{CONCLUSIONS}

The main contribution of this paper contains the following aspects. First of all, in order to optimize the reactive and active power in the distribution system with renewable energy and storage system, a dynamic optimal power flow problem is modelled by considering the conic relaxation of power flow equalities. And then, the model is decomposed after the introduction of the auxiliary variables about the junction buses. In the model, the convexity of feasible set enables the convergence of the decentralized algorithm. Finally, the decentralized algorithm based on the extended ADMM is proposed, such that the connected communication nodes exchange only the information they are interested in. The communication nodes work according their colours; thus an optimal solution can be obtained by use a minimal amount of communication. Simulations based on the 69-buses benchmark distribution system illustrate the effectiveness of 
the proposed decentralized optimization algorithm. Moreover, the effect of data packet dropout on the decentralized algorithm will be considered in our future work.

\section{REFERENCES}

[1] S. P. Singh, J. Rokadia, C. Mishra, "Optimal power flow in the presence of wind power using modified cuckoo search", IET Generation, Transmission \& Distribution, vol. 9, no. 7, pp. 615-626, 2015. DOI: 10.1049/iet-gtd.2014.0285.

[2] B. C. Pal, R. A. Jabr, "Intermittent wind generation in optimal power flow dispatching", IET Generation, Transmission \& Distribution, vol. 3, no. 1, pp. 66-74, 2009. DOI: 10.1049/iet-gtd:20080273.

[3] P. Faria, T. Soares, Z. Vale, H. Morais, "Distributed generation and demand response dispatch for a virtual power player energy and reserve provision", Renewable Energy, vol. 66, pp. 686-695, 2014. DOI: 10.1109/ISGTEurope.2012.6465760.

[4] A. Gopalakrishnan, A. U. Raghunathan, D. Nikovski, L. T. Biegler, "Global optimization of multi-period optimal power flow", in 2013 American Control Conf., 2013, pp. 1157-1164. DOI: 10.1109/ACC.2013.6579992.

[5] S. Gill, I. Kockar, G. W. Ault, "Dynamic optimal power flow for active distribution networks", IEEE Trans. Power Systems, vol. 29, no. 1, pp. 121-131, 2014. DOI: 10.1109/TPWRS.2013.2279263.

[6] A. J. Guwy, S. Carr, R. M. Dinsdale, J. Maddy, G. C. Premier, "Energy storage for active network management on electricity distribution networks with wind power", IET Renewable Power Generation, vol. 8 , no. 3, pp. 249-259, 2014. DOI: 10.1049/iet-rpg.2012.0210.

[7] W. Shi, N. Li, X. Xie, C.-C. Chu, R. Gadh, "Optimal residential demand response in distribution networks", IEEE Journal on Selected Areas in Communications, vol. 32, no. 7, pp. 1441-1450, 2014. DOI: 10.1109/JSAC.2014.2332131.

[8] S. Boyd, "Distributed optimization and statistical learning via the alternating direction method of multipliers", Foundations and Trends $®$ in Machine Learning, vol. 3, no. 1, pp. 1-122, 2010.

[9] R. Ramos de Souza, A. Roberto Balbo, L. Nepomuceno, E. Cassia Baptista, E. Martins Soler, R. Bento Nogueira Pinheiro, "A primal-dual interior/exterior point method, with combined directions and quadratic test in reactive optimal power flow problems", IEEE Latin America Transactions, vol. 15, no. 8, pp. 1413-1421, 2017. DOI: 10.1109/TLA.2017.7994787.

[10] C. Zhang, H. Chen, M. Guo, X. Wang, Y. Liu, D. Hua, "DC power flow analysis incorporating interval input data and network parameters through the optimizing-scenarios method", International Journal of Electrical Power \& Energy Systems, vol. 96, pp. 380-389, 2018. DOI: 10.1016/j.ijepes.2017.10.005.

[11] Q. Peng, S. H. Low, "Distributed optimal power flow algorithm for radial networks, I: balanced single phase case", IEEE Trans. Smart Grid, vol. 9, no. 1, pp. 111-121, 2018. DOI: 10.1109/TSG.2016.2546305

[12] M. Baradar, M. R. Hesamzadeh, "AC power flow representation in conic format", IEEE Trans. Power Systems, vol. 30, no. 1, pp. 546-547, 2015. DOI: 10.1109/TPWRS.2014.2326980.

[13] E. Dall'Anese, Hao Zhu, G. B. Giannakis, "Distributed optimal power flow for smart microgrids", IEEE Trans. Smart Grid, vol. 4, no. 3 , pp. 1464-1475, 2013. DOI: 10.1109/TSG.2013.2248175.

[14] M. Farivar, S. H. Low, "Branch flow model: relaxations and convexification-Part II", IEEE Trans. Power Systems, vol. 28, no. 3 , pp. 2565-2572, 2013. DOI: 10.1109/TPWRS.2013.2255318.

[15] J. Li, W. Wei, "Probabilistic evaluation of available power of a renewable generation system consisting of wind turbines and storage batteries: A Markov chain method", Journal of Renewable and Sustainable Energy, vol. 6, no. 1, p. 013139, 2014. DOI: $10.1063 / 1.4866259$

[16] J. Li, W. Wei, J. Xiang, "A simple sizing algorithm for stand-alone $\mathrm{PV} /$ wind/battery hybrid microgrids", Energies, vol. 5, no. 12 pp. 5307-5323, 2012. DOI: 10.3390/en5125307.

[17] D. S. Wicaksono, I. A. Karimi, "Piecewise MILP under- and overestimators for global optimization of bilinear programs", AIChE Journal, vol. 54, no. 4, pp. 991-1008, 2008. DOI: 10.1002/aic.11425.

[18] L. Collins, J. K. Ward, "Real and reactive power control of distributed $\mathrm{PV}$ inverters for overvoltage prevention and increased renewable generation hosting capacity", Renewable Energy, vol. 81, pp. 464-471, 2015. DOI: 10.1016/j.renene.2015.03.012.

[19] J. F. C. Mota, J. M. F. Xavier, P. M. Q. Aguiar, M. Puschel, "Distributed optimization with local domains: applications in MPC and network flows", IEEE Trans. Automatic Control, vol. 60, no. 7 , pp. 2004-2009, 2015. DOI: 10.1109/TAC.2014.2365686.

[20] J. Li, H. Xin, W. Wei, W. Dai, "Decentralised conic optimisation of reactive power considering uncertainty of renewable energy sources", IET Renewable Power Generation, vol. 10, no. 9, pp. 1348-1355, 2016. DOI: 10.1049 /iet-rpg.2015.0172.

[21] J. Li, H. Xin, W. Wei, W. Dai, "Communication-efficient distributed strategy for reactive power optimisation considering the uncertainty of renewable generation", IET Generation, Transmission \& Distribution, vol. 10 , no. 15, pp. 3681-3690, 2016. DOI 10.1049/iet-rpg.2015.0172.

[22] D. Janulevicius, A. Valinevicius, D. Andriukaitis, "Intelligent plug-in hybrid electric vehicle", in Proc. of the 7th international scientific conference and 4th international symposium of young researchers Transport problems, Katowice, Poland, 2015, pp. 215-226.

[23] C. Wu, F. Wen, Y. Lou, F. Xin, "Probabilistic load flow analysis of photovoltaic generation system with plug-in electric vehicles", International Journal of Electrical Power \& Energy Systems, vol. 64, pp. 12211228, 2015. DOI: 10.1016/j.ijepes.2014.09.014. 